\title{
Neural Correlates of Syntactic Ambiguity in Sentence Comprehension for Low and High Span Readers
}

\author{
Christian J. Fiebach ${ }^{1}$, Sandra H. $\operatorname{Vos}^{2,3}$, and Angela D. Friederici ${ }^{1}$
}

\begin{abstract}
Syntactically ambiguous sentences have been found to be difficult to process, in particular, for individuals with low working memory capacity. The current study used fMRI to investigate the neural basis of this effect in the processing of written sentences. Participants with high and low working memory capacity read sentences with either a short or long region of temporary syntactic ambiguity while being scanned. A distributed left-dominant network in the peri-sylvian region was identified to support sentence processing in the critical region of the sentence. Within this network, only the superior portion of Broca's area (BA 44) and a parietal region showed an acti-
\end{abstract}

\section{INTRODUCTION}

The cognitive mechanisms of sentence processing are constrained by the working memory resources of the cognitive system which are limited in their capacity for storage and computational operations (e.g., Caplan \& Waters, 1999; Just \& Carpenter, 1992). One of the most influential paradigms employed in psycholinguistics for studying the resource usage during sentence processing is the investigation of how temporary syntactic ambiguities such as [1] (taken from MacDonald, Just, \& Carpenter, 1992) are resolved by the sentence processor.

[1] The experienced soldiers warned about the dangers ...

In a sentence like [1] the verb "warned" may either be the main verb as in The experienced soldiers warned about the dangers before the midnight raid, in which case "soldiers" is the agent of the sentence. Alternatively, the verb "warned" may introduce a relative clause, in which case "soldiers" is the patient of "warned" rather than the agent, as in The experienced soldiers warned about the dangers conducted the midnight raid.

Research on ambiguity resolution has yielded evidence that working memory resources are critical for maintaining alternative interpretations of a sentence

\footnotetext{
${ }^{1}$ Max Planck Institute of Cognitive Neuroscience, Germany,

${ }^{2}$ F. C. Donders Centre for Cognitive Neuroimaging, Nijmegen,

${ }^{3} \mathrm{Nijmegen} \mathrm{Institute} \mathrm{for} \mathrm{Cognition} \mathrm{and} \mathrm{Information}$
}

vation increase as a function of the length of the syntactically ambiguous region in the sentence. Furthermore, it was only the BA 44 region that exhibited an interaction of working memory span, length of the syntactic ambiguity, and sentence complexity. In this area, the activation increase for syntactically more complex sentences became only significant under longer regions of ambiguity, and for low span readers only. This finding suggests that neural activity in BA 44 increases during sentence comprehension when processing demands increase, be it due to syntactic processing demands or by an interaction with the individually available working memory capacity.

available in working memory during the ambiguous region of the sentence, a strategy that is favorable for later resolving the ambiguity (Mitchell, 1994; MacDonald et al., 1992). However, it is to date not fully solved which precise role individual differences in working memory resources play during this process. Some studies suggest that individuals with a greater working memory capacity are better in maintaining alternative readings than individuals with less resources (e.g., MacDonald et al., 1992). Other reports indicate the opposite, namely, that high span readers are more efficient in inhibiting a dispreferred reading, whereas low span individuals use a more resource-demanding strategy of maintaining multiple alternative readings in working memory (Friederici, Steinhauer, Mecklinger, \& Meyer, 1998).

The neural bases of such working memory processes during sentence processing are only beginning to be understood in more detail. Several functional neuroimaging studies using positron emission tomography (PET) or functional magnetic resonance imaging (fMRI) have investigated the neural bases of syntactic processing (see Friederici, 2002; Kaan \& Swaab, 2002, for recent reviews). Most studies (e.g., Ben-Schachar, Hendler, Kahn, Ben-Bashat, \& Grodzinsky, 2003; Caplan, Vijayan, et al., 2002; Röder, Stock, Neville, Bien, \& Rösler, 2002; Caplan, Alpert, Waters, \& Oliveri, 2000; Caplan, Alpert, \& Waters, 1998, 1999; Just, Carpenter, Keller, Eddy, \& Thulborn, 1996; Stromswold, Caplan, Alpert, \& Rauch, 1996) focused on investigating the neuroanatomical correlates of syntactic complexity effects. A common 
finding of these studies is that structurally complex sentences elicit greater activity than less complex sentences in the left fronto-opercular region, in particular, in the posterior portion of the inferior frontal gyrus (IFG) (i.e., in Broca's area). However, early studies in this field could not make specific conclusions regarding the underlying mechanisms of these complexity effects as they could not dissociate syntactic complexity from working memory demands arising during the processing of sentences of different complexity (e.g., Just et al., 1996; Stromswold et al., 1996). More recently, it was suggested that complexity effects observed in Broca's area might also be due to working memory costs induced when processing structurally complex sentences (cf. Cooke et al., 2001; Fiebach, Schlesewsky, \& Friederici, 2001).

A more direct way to test the assumption of an involvement of Broca's area in syntactic working memory processes is to explore activity of this area during the processing of sentences with a temporary syntactic ambiguity. Earlier studies investigated the processing of syntactically ambiguous sentences using event-related brain potentials (ERPs) in individuals with high or low working memory capacity (Vos, Gunter, Schriefers, \& Friederici, 2001; Friederici et al., 1998; Mecklinger, Schriefers, Steinhauer, \& Friederici, 1995). In these studies, a detailed understanding of the temporal dynamics of resolving syntactic ambiguities could be gained. It was demonstrated that positive-going ERP components are elicited when temporarily ambiguous sentences are disambiguated towards an unpreferred interpretation. This ERP effect is modulated by the length over which the syntactic ambiguity persists, as well as by individual differences in working memory capacity (Friederici et al., 1998). The ERP data indicate that both the total capacity that is available and the efficiency of utilizing that capacity are critical for syntactic ambiguity resolution (Vos, Gunter, Schriefers, et al., 2001). For the purposes of identifying the brain bases of syntactic ambiguity resolution, we adapted the psycholinguistic paradigm used in these ERP studies, and conducted an fMRI study at $3 \mathrm{~T}$ in which we manipulated the time point of disambiguation in the sentences and thereby the length of the ambiguous region, as well as the individual working memory capacity.

\section{The Present Study}

Two groups of subjects were preselected, one group of individuals with low verbal working memory capacity and a high capacity group, as assessed with the Daneman and Carpenter (1980) reading span test. Daneman and Carpenter demonstrated that this verbal working memory test draws simultaneously on storage and computational aspects of working memory. They further demonstrated that both the reading span test and an auditory version of the test, the listening span, correl- ated better with sentence comprehension than more traditional measures of working memory based on storage alone, such as the digit span test (see also Daneman \& Merikle, 1996; Waters \& Caplan, 1996). Since its introduction, the reading span test has become the standard measure to assess individual working memory capacity in the context of sentence processing.

The present study investigated the potential effect of different sentence processing demands on brain activity by using sentence material of various processing costs along two dimensions. It is well known that the more common subject-first word-order in German relative clauses is easier to interpret and understand than the more demanding object-first word-order (e.g., Friederici, Steinhauer, et al., 1998; Schriefers, Friederici, \& Kuehn, 1995; Frazier, 1987). A second well-known sentential manipulation on verbal memory capacity is the length of the ambiguous region of the sentence: In short, the longer the region, the more capacity is required (e.g., MacDonald et al., 1992; see also Friederici, Steinhauer, et al., 1998). These two sentential manipulations-word order and length of ambiguity — can be perfectly crossmanipulated in German relative clauses, as demonstrated in Table 1.

Table 1 presents examples of the four types of temporarily ambiguous sentences used in the present study [examples are given in German (a), in a direct word-byword translation into English (b), and in a free English translation (c)]. All sentences are relative clauses which are temporarily ambiguous up to the presentation of the underlined word.

There are three features of German syntax of relevance for this study. The first is that, in contrast to English, the finite verb of the subordinate clause ("have") occurs in clause-final position. Second, subject and verb agree in number in German. Third, in German, the feminine relative pronoun "die" is used in both the nominative and accusative forms for singular and plural, as well as in the masculine plural form. These properties allow us to construct the four sentence conditions in Table 1 . In the late-disambiguation conditions (1) and (2), sentences are disambiguated by the number-information of the clause-final auxiliary verb. That is, until the presentation of this clause-final verb, the German sentences (1a) and (2a) are ambiguous between an SR (subject relative) and an OR (object relative) reading. In the SR structure (1a), there is number agreement between the auxiliary and the first noun-phrase (i.e., "die Sportlerin"), while in the OR structure (2a), there is number agreement between the auxiliary and the second noun-phrase (i.e., "die Trainerinnen"). Notice that this rather long region of ambiguity with respect to an SR or OR reading differs from the English translations (1c) and (2c) that are only temporarily ambiguous from the beginning of the sentences up to and including "who."

Sentences in the early-disambiguation conditions (3) and (4) are disambiguated by case-marking at the sec- 
Table 1. Experimental Sentence

\section{Late disambiguation, subject relative:}

a. Das ist die Sportlerin (feminine, singular), die die Trainerinnen (feminine, plural) gesucht hat (singular), weil der Diskus feblte.

b. This is the athlete who the trainers looked for has, because the discus got lost.

c. This is the athlete who looked for the trainers, because the discus got lost.

2. Late disambiguation, object relative:

a. Das ist die Sportlerin (feminine, singular), die die Trainerinnen (feminine, plural) gesucht haben (plural), weil der Diskus feblte.

b. This is the athlete who the trainers looked for have, because the discus got lost.

c. This is the athlete who the trainers looked for, because the discus got lost.

\section{Early disambiguation, subject relative:}

a. Das sind die Einwohner (masculine, plural), die den Polizisten (masculine, singular, accusative) angehört baben (plural), weil in den Garten eingebrochen worden war.

b. This are the inhabitants who the policeman listened to have, because into the garden broken in was.

c. These are the inhabitants who listened to the policemen, because the garden was broken in into.

\section{Early disambiguation, object relative:}

a. Das sind die Einwohner (masculine, plural), die der Polizist (masculine, singular, nominative) angebört hat (singular), weil in den Garten eingebrochen worden war.

b. This are the inhabitants who the policeman listened to has, because into the garden broken in was.

c. These are the inhabitants to whom the policeman listened, because the garden was broken in into.

The examples are given in original German (a), a direct word-by-word translation into English (b), and a free English translation (c).

ond noun-phrase of the relative clause. That is, in contrast to the feminine nouns used for the late-disambiguation condition, the masculine singular noun phrases do differ in the nominative and accusative forms. As a result, in (3a) and (4a) the sentences are disambiguated by case-marking of the determiners "der" and "den" to either an SR or an OR structure. Sentences of these four types were presented visually to two groups of participants, one group consisting of individuals with a high working memory span, the other of individuals with a low working memory span. During the fMRI scanning session, the participants had the task to verify the content of a probe sentence presented after the critical sentences. This behavioral task required that participants resolved the ambiguity present in the critical sentences.

Three potential effects are of main interest for the present study. First, it is expected that brain activation should vary as a function of working memory load in the ambiguous regions of the sentences. Late disambiguated sentences should display greater activity than early disambiguated sentences because of the necessity to maintain multiple possible readings of the sentences over a prolonged region of the sentence. This prediction concerns the time interval between the disambiguation points of early and late disambiguated sentences. Second, hemodynamic correlates of transient ERP effects elicited at the disambiguating element (see above) might be observable at the disambiguating word itself. Third, based on the ERP data cited above, we expected to find a dependency of some of these effects on individual working memory capacity. For this reason, statistical analyses were conducted such that interactions between the factor span group and the two withinsubjects factors, disambiguation time point and word order, could be examined.

\section{RESULTS}

\section{Behavioral Responses}

The analysis of the reaction times in a $2 \times 2$ factorial design yielded a reliable main effect of disambiguation point $[F(1,14)=7.68 ; p<.05]$ and a significant effect of word order $[F(1,14)=4.68 ; p<.05]$. Late disambiguated sentences and object-initial relatives induced longer response times (cf. Table 2a). There was no interaction effect between the two factors $(F<.5)$. This pattern was partly reflected in the percentage of correctly answered trials, which also showed a significant main effect of disambiguation point $[F(1,14)=16.42 ; p<.005]$ but no effect of word order $[F(1,14)=1.17 ; p=.3]$ and also no interaction $(F<.5)$.

When considering the between-subjects factor reading span, we observed a tendency towards faster responses in high span individuals $[F(1,13)=3.88 ; p=.07]$, but no interactions between span group and any of the syntactic factors in the response times (see Table 2b). In the percentage of correct responses, no main effect of span group was seen $(F<1)$, but a reliable interaction of span group and disambiguation point $[F(1,13)=11.12$; $p=.005]$. This interaction was due to a significant effect of the time point of disambiguation only in the low span group $[F(1,7)=27.3 ; p=.001]$.

Thus, the behavioral data showed that overall participants had more difficulty in comprehending object-first structures in comparison to subject-first structures, and more difficulty in comprehending late disambiguating structures in comparison to early disambiguating ones. 
Table 2.

\begin{tabular}{|c|c|c|c|c|c|c|c|c|}
\hline & \multicolumn{3}{|c|}{ SR, Early } & OR, Early & \multicolumn{3}{|c|}{ SR, Late } & OR, Late \\
\hline Reaction times (msec) & \multicolumn{3}{|c|}{$841.1(47.0)$} & $871.3(49.9)$ & \multicolumn{3}{|c|}{$890.6(58.1)$} & $910.0 \quad(55.2)$ \\
\hline Percent correct & \multicolumn{3}{|c|}{$95.4 \%(1.5)$} & $94.2 \%(1.2)$ & \multicolumn{3}{|c|}{$90.2 \%(1.9)$} & $88.5 \%(2.5)$ \\
\hline \multicolumn{9}{|c|}{$\begin{array}{l}\text { b. RTs and Percentage of Correct Responses for the Sentence Verification Task for Low Span }(n=8) \text { and High Span }(n=7) \\
\text { Participants }\end{array}$} \\
\hline & \multicolumn{4}{|c|}{ Low Span } & \multicolumn{4}{|c|}{ High Span } \\
\hline & $S R$, Early & OR, Early & SR, Late & OR, Late & SR, Early & OR, Early & SR, Late & OR, Late \\
\hline Reaction times (msec) & $931.4(59.4)$ & $953.4(64.1)$ & $979.7(77.5)$ & $993.8(71.2)$ & $737.8(54.8)$ & $777.4(64.8)$ & $788.7(74.9)$ & $814.2(74.9)$ \\
\hline Percent correct & $96.1 \%(1.8)$ & $94.1 \%(1.6)$ & $88.3 \%(2.4)$ & $84.8 \%(3.9)$ & $94.6 \%(2.5)$ & $94.2 \%(1.9)$ & $92.4 \%(3.0)$ & $92.9 \%(2.1)$ \\
\hline
\end{tabular}

Note: The percentages refer to the percentage of correct answers. Standard errors are presented between parentheses.

$\mathrm{SR}=$ subject relative; $\mathrm{OR}=$ object relative; early and late refer respectively to early and late disambiguation.

This latter effect was particularly evident in low span subjects.

\section{Functional Imaging Data}

Brain activation results are reported in two steps. First, we describe those areas which showed reliably increased activation in the time interval between the early and late disambiguation points, independent of the syntactic manipulations applied in the present study. This activation was identified by determining the departure of the hemodynamic response from the overall mean of the whole time series, thus isolating brain regions which were particularly involved in processing the critical region of the experimental sentences. We then examine whether or not activity in these brain areas was modulated by word order (i.e., SR vs. OR) or by the point at which the temporarily ambiguous sentences were resolved towards one of the two possible readings (i.e., early vs. late point of disambiguation). For both analyses, we first report results for the whole group, and then compare individuals with high and low working memory capacity.

\section{Sentence Processing}

During the critical period of the sentences, analyzed by modeling an epoch of $2.5 \mathrm{sec}$ length (see Methods section), reliable brain activation was observed in an extended peri-sylvian network, including the area around the posterior superior temporal sulcus and the middle temporal gyrus (MTG) (i.e., Wernicke's area), the anterior superior temporal gyrus (STG), and superior temporal sulcus (STS), as well as the left fronto-opercular region (Figure 1A). The latter activation cluster encompassed Brodmann's areas (BA) 44 and 45 laterally, as well as cortical tissue more deeply in the frontal operculum and in the anterior insula. In the right hemisphere, homologous activity was found in the posterior superior temporal sulcus, in the posterior third of the IFG, and in the anterior insula. Furthermore, brain activation related to sentence processing was also seen in the basal ganglia (caudate nucleus, putamen, and globus pallidus) and in the thalamus. Finally, we also observed activity in the posterior portion of the intraparietal sulcus in both hemispheres. Talairach and Tournoux (1988) coordinates for the reported brain areas, as well $Z$-values at the peak voxels are listed in Table 3. For activation clusters which encompassed multiple functionally significant areas, local maxima were determined within the activated clusters.

Separate analyses for low span $(n=8)$ and high span $(n=7)$ individuals appear to suggest that the perisylvian network described above is more strongly activated for low span individuals than for high span individuals (Figure 1B and C). However, by submitting the individual contrast images to a whole-brain, voxelwise, two-sample $t$ test testing for between-group differences, we could not identify span group differences in the exact brain regions described above for processing the critical region of the sentences. This direct comparison of the two groups, however, indicated three activation clusters outside of the above-described network for sentence processing, in which low span individuals showed greater activation than high capacity individuals (Figure 1D). These regions were the left inferior-most part of the anterior insula, the right anterior- and inferior-most basal ganglia, encompassing ventral portions of the head of the caudate nucleus and the putamen, as well as the anterior and medial portion of the right thalamus. There were also three areas activated 


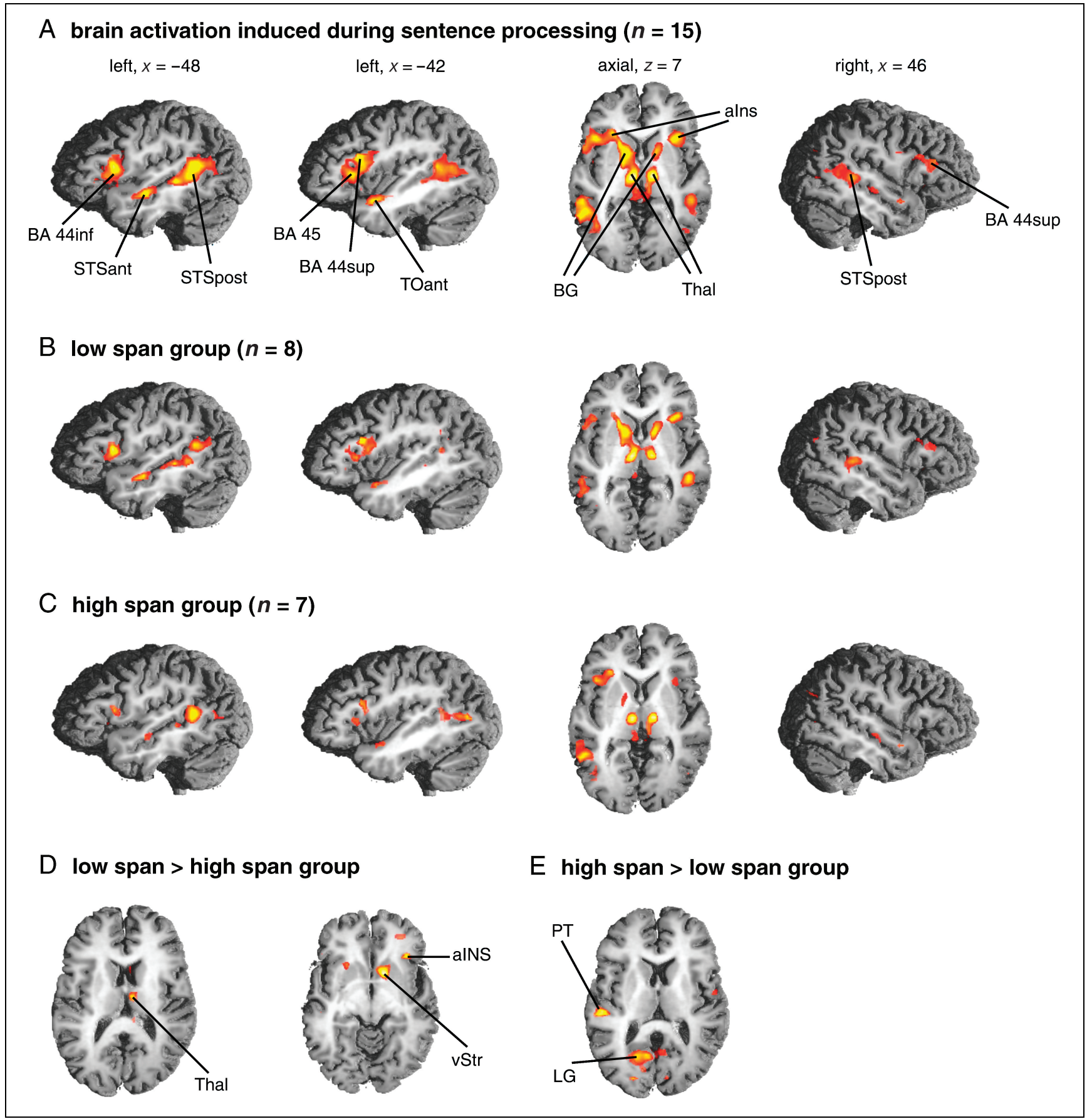

Figure 1. Brain activation for sentence processing for all participants (A) and analyzed separately for the low (B) and high (C) span groups. Activation in the intraparietal sulcus (see Table 3 ) is not shown. The direct comparison between the groups revealed greater activity for low than for high span participants (D) in the left inferior-anterior insula (not shown; $x=-23, y=18, z=3 ; z_{\max }=3.01 ; 27$ voxels; $p_{\text {clustersize }}=.0025$ ), the right ventral striatum $\left(10,9,0 ; z_{\max }=3.68 ; 55\right.$ voxels; $\left.p_{\text {cluster }}=.003\right)$, and in the right thalamus $\left(7,-15,18 ; z_{\max }=3.46 ; 30\right.$ voxels; $\left.p_{\text {cluster }}=.019\right)$. High span participants showed greater activity than low span participants (E) in the cuneus $\left(10,-90,29 ; z_{\max }=3.74 ; 62\right.$ voxels; $\left.p_{\text {cluster }}=.002\right)$, the left lingual gyrus $\left(-17,-63,9 ; z_{\max }=3.25 ; 129\right.$ voxels; $\left.p_{\text {cluster }}<.000\right)$, and in the left planum temporale $\left(-53,-27,15 ; z_{\max }=3.52 ; 22\right.$ voxels; $\left.p_{\text {cluster }}=.040\right)$. (A-C) Statistical parametric maps thresholded at $z>3.09(p<.001)$; (D-E) Statistical parametric maps thresholded at $p<.01$ (see Methods section). BA 44inf $=$ inferior portion of BA 44; BA 44sup = superior portion of BA 44; STSant $=$ anterior superior temporal sulcus; STSpost $=$ posterior superior temporal sulcus; TOant $=$ temporal operculum, anterior portion; $\mathrm{BG}=$ basal ganglia; $\mathrm{vStr}=\mathrm{ventral}$ striatum; Thal $=$ thalamus; aINS $=$ anterior insular cortex; PT $=$ planum temporale; LG $=$ lingual gyrus .

more strongly for individuals with high working memory capacity, namely, the cuneus superior to the calcarine sulcus, the left lingual gyrus, and an area in the left planum temporale immediately posterior to the auditory cortex (Figure 1E; see figure caption for Talairach and Tournoux [1988] coordinates). 
Table 3. Functional Imaging Results. Local Maxima of Brain Regions Activated during Sentence Processing

\begin{tabular}{|c|c|c|c|c|c|c|}
\hline & \multirow[b]{2}{*}{ Hemisphere } & \multirow[b]{2}{*}{ Brodmann's Area } & \multirow[b]{2}{*}{$Z_{\max }$} & \multicolumn{3}{|c|}{ Coordinates $^{\mathrm{a}}$} \\
\hline & & & & $x$ & $y$ & $z$ \\
\hline Inferior frontal gyrus (pars opercularis) & $\mathrm{L}$ & 44 & 5.67 & -46 & 12 & 14 \\
\hline \multirow[t]{2}{*}{ Inferior frontal gyrus (superior portion) } & $\mathrm{L}$ & $44 / 6$ & 5.04 & -40 & 15 & 23 \\
\hline & $\mathrm{R}$ & & 4.25 & 43 & 12 & 23 \\
\hline Inferior frontal gyrus (pars triangularis) & $\mathrm{L}$ & 45 & 4.69 & -40 & 18 & 8 \\
\hline \multirow[t]{2}{*}{ Anterior insula } & $\mathrm{L}$ & & 4.83 & -25 & 24 & 2 \\
\hline & $\mathrm{R}$ & & 4.81 & 28 & 18 & 5 \\
\hline \multirow[t]{2}{*}{ Basal ganglia (head of caudate, putamen, globus pallidus) } & $\mathrm{L}$ & & 4.56 & -13 & 9 & 2 \\
\hline & $\mathrm{R}$ & & 3.95 & 16 & 9 & 5 \\
\hline \multirow[t]{2}{*}{ Thalamus } & $\mathrm{L}$ & & 5.82 & -10 & -17 & 11 \\
\hline & $\mathrm{R}$ & & 5.32 & 10 & -14 & 8 \\
\hline Superior temporal gyrus/sulcus (antero-lateral portion) & $\mathrm{L}$ & $22 / 38$ & 4.71 & -49 & -11 & -5 \\
\hline Superior temporal gyrus/sulcus (antero-medial portion) & $\mathrm{L}$ & $22 / 38$ & 4.56 & -40 & 3 & -11 \\
\hline \multirow[t]{2}{*}{ Superior temporal sulcus/middle temporal gyrus (posterior) } & $\mathrm{L}$ & $21 / 37$ & 4.95 & -52 & -53 & 14 \\
\hline & $\mathrm{R}$ & & 4.18 & 52 & -44 & 17 \\
\hline \multirow[t]{2}{*}{ Intraparietal sulcus } & $\mathrm{L}$ & $39 / 7 b$ & 5.13 & -28 & -65 & 41 \\
\hline & $\mathrm{R}$ & & 4.24 & 31 & -62 & 41 \\
\hline
\end{tabular}

${ }^{\mathrm{a}}$ Coordinates given represent Talairach and Tournoux (1988) coordinates.

\section{Word Order and Disambiguation Point}

When the effects of the syntactic manipulations were analyzed in a region-of-interest (ROI) analysis (see Methods) for the brain areas listed in Table 3, none of the ROIs showed a main effect of word order. A main effect of disambiguation time point was seen in the superior portion of BA 44 bilaterally $[F(1,14)=8.4 ; p<.025$; Figure $2 \mathrm{~A}]$ and in the intraparietal sulcus bilaterally $[F(1,14)=7.6 ; p<.025$; Figure $2 \mathrm{~B}]$. In both regions, late disambiguation elicited greater activity than early disambiguation. Disambiguation effects did not differ between hemispheres (interactions $F<1$ ).

In addition, we observed several subcortical effects. In the thalami, we detected an interaction of hemisphere with word order $[F(1,14)=9.4 ; p<.01]$ which was due to the fact that the left thalamus showed a tendency for stronger activation for object-first as compared to subject-first sentences $(p=.083)$, whereas the right thalamus showed the reverse pattern $(p=.082)$. In the basal ganglia, there were reliable interactions between hemisphere and word order $[F(1,14)=7.4 ; p<.025$; Figure $2 \mathrm{C}]$ and between hemisphere and disambiguation point $[F(1,14)=8.2 ; p<.025$; Figure $2 \mathrm{D}]$. These interactions, however, could not be resolved to show clear interhemispheric differences.
A significant interaction involving individual working memory span was seen only in one area, namely, in the superior portion of BA 44. Here, an interaction of span group, disambiguation point, and word order was seen independent of hemisphere $[F(1,13)=6.3 ; p=.026]$. This interaction could be resolved to show greater activity for late disambiguated OR as compared to SR sentences only in the low span group $[F(1,7)=9.3 ; p<$ .025; Figure 2E]. No effect was observed for the high span group (Figure 2F). Moreover, no effects were seen in the early disambiguated sentences. No further interactions involving span group or main effects of span group became significant.

In the present analysis, in which we modeled the time interval between the two disambiguation points as an epoch (i.e., a box-car function convolved by a canonical HRF function), it might be the case that transient effects taking place at the disambiguation point itself might not be captured. This might be so because the modeled epoch encompasses $2.5 \mathrm{sec}$ of sentence processing, whereas certain processes very likely happen in the order of a few hundred milliseconds. As transient effects reflecting parsing processes associated with ambiguity resolution are well documented in the ERP literature (Vos, Gunter, Schriefers, et al., 2001; Friederici, Steinhauer, et al., 1998; Mecklinger et al., 1995), we 
Figure 2. Mean contrast values for the ROIs showing a significant main effect of disambiguation point (superior BA 44 and intraparietal sulcus), interactions of hemisphere and condition factors (basal ganglia), and an interaction of working memory span, disambiguation point, and word order (superior BA 44)

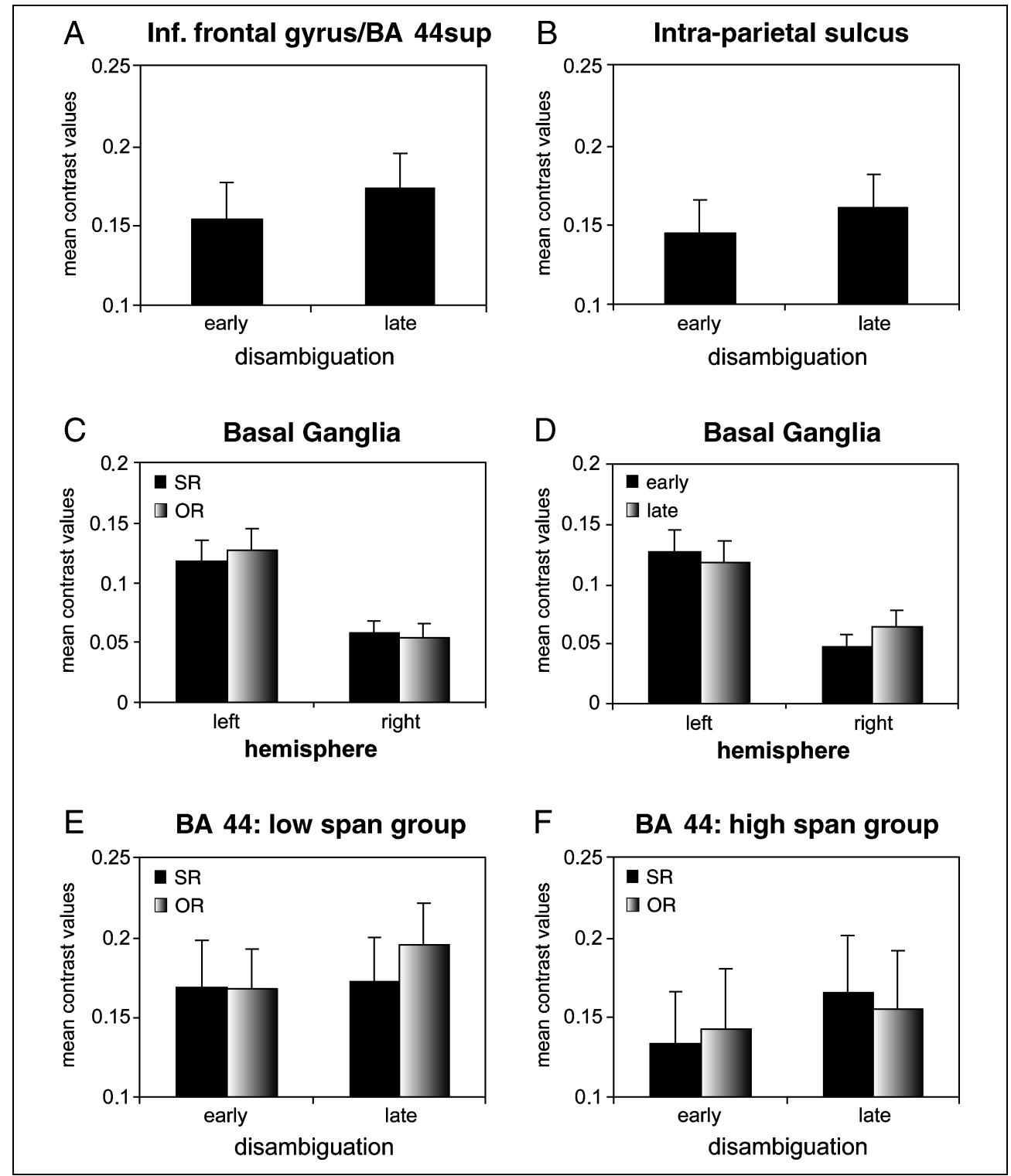

performed an additional analysis in which we modeled as a critical event the time point at which sentences were disambiguated towards a subject-first or objectfirst reading. In this analysis, the events modeled in early and late disambiguated sentences were separated by $2.5 \mathrm{sec}$, which might pose a problem for directly comparing early and late disambiguated sentences. For this reason, we used this analysis exclusively for comparing OR and SR sentences, independent of the time point of disambiguation. We reasoned that this eventrelated approach might be more sensitive to activation differences associated with word order, given the problems outlined above. However, this analysis resulted in the same pattern of brain activation effects as those obtained in the epoch-based analysis (i.e., no reliable effects of word order in any of the ROIs examined were observed).

\section{DISCUSSION}

The present study set out to specify the neural correlates of the behaviorally well-established finding that individual working memory capacity interacts with sentence comprehension. A distributed network of perisylvian and subcortical regions was identified as being involved in sentence processing during the relative clause region in individuals with a high as well as those with a low working memory span. Within this network, the superior portion of BA 44 and a parietal region showed greater activity for late than for early disambiguated sentences. This effect is a neural correlate of the behavioral effect of disambiguation time point, which showed slower reaction times and more errors for late disambiguated sentences. In the brain imaging data, the superior BA 44 also showed an interaction involving the 
between-group factor span. When processing late disambiguated sentences, only low span individuals showed an activation increase in this area for sentences disambiguated towards a dispreferred OR reading.

In the present study, we used written sentence material and a German adaptation of the classical Daneman and Carpenter (1980) span test which also involved written sentence presentation. Although the syntactic mechanisms targeted by the present study are assumed to be modality independent, it is interesting to speculate whether different effects would have been obtained when an auditory span task was combined with auditory sentence presentation. At hand, there is no functional neuroimaging study reported investigating this issue. However, it is well known that both the reading version and the listening version of the Daneman and Carpenter task correlate well with sentence processing. Furthermore, there is an ERP study that used a listening span task and found interactions with visual sentence processing (Vos, Gunter, Kolk, \& Mulder, 2001), supporting the assumption that the processes investigated here are not influenced by the modality of sentence presentation.

In the following, the findings of the current study will be discussed against the background of other studies reporting activity in BA 44 during sentence processing. Before that, we will briefly consider the network of brain areas identified in the present study as being involved in reading of sentences during the relative clause region.

\section{The Cortical and Subcortical Involvement in Sentence Processing}

Similar to earlier imaging studies, we observed activity in a large, left-dominant temporo-frontal cortical network, as well as an involvement of subcortical structures, when we compared the hemodynamic responses elicited during the critical portion of the relative clause sentences to a baseline representing the average signal of the acquired time series. The cortical areas observed here have been reported in several previous studies (for recent reviews, see Friederici, 2002; Kaan \& Swaab, 2002). The temporal part of the language-related network includes the antero-lateral, antero-medial, and posterior portions of the STG extending into the STS. The anterior portion of the STG is known to support sentence comprehension (e.g., Humphries, Kimberley, Buchsbaum, \& Hickok, 2001), in particular, syntactic processes (Friederici, Rueschemeyer, Hahne, \& Fiebach, 2003; Friederici, Meyer, \& von Cramon, 2000; Meyer, Friederici, \& von Cramon, 2000). In the domain of auditory processing, activation in the anterior STS was shown to vary as a function of speech intelligibility (Scott, Blank, Rosen, \& Wise, 2000). The posterior portion of the STS and the MTG have been associated primarily with semantic processes (Kotz, Cappa, von Cramon, \& Friederici, 2002; Ni et al., 2000; Dapretto \& Bookheimer, 1999;
Pugh et al., 1996; Kapur et al, 1994). The role of the prefrontal component of the cortical language network will be discussed in more detail below.

At the subcortical level, the thalamus and the basal ganglia demonstrated increased activation during reading of sentences. The left thalamus was somewhat more activated for object-first as compared to subject-first sentences although this was only observed as a trend. Earlier studies on syntactic processing also reported activation in the left thalamus (Luke, Liu, Wan, \& Tan, 2002; Kuperberg et al., 2000). Activation in the basal ganglia had been found as a function of syntactic processing in imaging studies as well. The left caudate nucleus was observed to be activated during sentence processing in general (Kuperberg et al., 2000), and during syntactic processing in particular (Moro et al., 2001). The left putamen was also reported to activate as a function of syntactic processing (Friederici, Rueschemeyer, et al., 2003). Patient studies support the view that the basal ganglia are involved in syntactic processing: Parkinson's patients suffering from a degeneration of basal ganglia functions have been shown to deficiently perform on syntactic production tasks (Ullman et al., 1997) and in syntactic comprehension (Grossman et al., 1991). Moreover, patients with Parkinson's disease and with lesions in the basal ganglia differ in their ERP pattern from healthy subjects during syntactic processing (Friederici, Kotz, Werheid, Hein, \& von Cramon, 2003; Kotz, Frisch, von Cramon, \& Friederici, 2003). The basal ganglia, most generally, have been taken to play an important role in action selection (Bergman et al., 1998), or possibly in the effective inhibition of alternative meanings (Copland, Chenery, \& Murdoch, 2001). The latter function is crucial when processing ambiguous sentences, as at some point in the comprehension process a selection of one of the two possible interpretations must be made. It has been proposed that high span readers are more successful in sentence interpretation due to a more effective inhibition of the dispreferred reading during sentence comprehension, whereas low span readers try to keep the two alternative readings active in working memory (Friederici, Steinhauer, et al., 1998; see also Gunter, Wagner, \& Friederici, 2003, for converging evidence from lexical ambiguity resolution). This latter notion receives support from the finding that activation in the right basal ganglia is stronger in low than in high span participants. This interpretation of the span group effect seen in the right basal ganglia, however, rests on the assumption of an additional recruitment of right hemispheric areas under conditions of increased processing demands in language comprehension.

Taken together, the activations observed in lateral cortical regions are compatible with earlier findings for sentence processing. Basal ganglia activation in the present study might be related to inhibitory processes necessary for the effective processing of ambiguous 
sentences, a demand that is more difficult for individuals with low compared to high working memory capacity.

\section{The Role of the Left Inferior Frontal Cortex in Sentence Processing}

The frontal part of the cortical network identified here encompasses large portions of the IFG-namely, its superior posterior portion, the pars opercularis, and the pars triangularis-as well as the anterior insula. The left IFG regions have-among other cognitive processes such as the articulatory rehearsal component of verbal working memory (e.g., Smith \& Jonides, 1999) or phonological processing (e.g., Poldrack et al., 1999)— been associated with syntactic processing (Ben-Schachar et al., 2003; Suzuki \& Sakai, 2003; Röder et al., 2002; Caplan, 2001; Embick, Marantz, Miyashita, O'Neil, \& Sakai, 2000; Friederici, Meyer, et al., 2000), whereby the pars triangularis appears to be less specific for syntactic processes as this area in many studies was also found to subserve semantic processes (see Bookheimer, 2002). The involvement of BA 44 in syntactic processing has also been demonstrated in a number of recent studies. Although some suggest that BA 44 is specifically responsible for syntactic processes (Ben-Shachar et al., 2003; Embick et al., 2000), others take their data to support the view that BA 44 comes into play when syntactic processes become more demanding not only in terms of necessary syntactic transformations but, moreover, when memory-demanding dependencies between two elements in a sentence must be established (Fiebach, Schlesewsky, Lohmann, von Cramon, \& Friederici, 2005; Cooke et al., 2001; Fiebach, Schlesewsky, \& Friederici, 2001). Fiebach et al. investigated the processing of object-first and subject-first wh-sentences and varied the distance between the topicalized element (wh-word) and the position from which it was moved (its so-called trace). They found that not the differences in the word order (i.e., object- vs. subject-first) alone determined activation in BA 44, but rather the length of the distance between the topicalized (moved) element and its original position, and the working memory demands resulting from this dependency.

So far, only one functional imaging study investigated the effect of individual working memory capacity during sentence processing (Waters, Caplan, Alpert, \& Stanczak, 2003). In this study, the influence of working memory span on word-order manipulations similar to the ones used in the present study (i.e., subject-first vs. objectfirst relative clauses) was investigated. Consistent with the present results, Waters et al. (2003) did not observe a two-way interaction of memory span group and word order in brain activation, neither in BA 44 nor in any other brain area. Based on these findings, the authors concluded that brain activation elicited during sentence processing is not dependent upon individual working memory capacity. The present results, in contrast, sug- gest that the amount of individually available working memory capacities does influence how the brain processes sentences, at least in memory taxing sentences such as those used in the present study. One reason for the difference between the study by Waters et al. and the present study most likely is the fact that the English relatives used by Waters et al. are disambiguated (by word order) relatively early in the sentences, making them more comparable to the early disambiguation than to the memory-taxing late disambiguation conditions of the present study. Converging evidence for this conclusion comes from a recent electrophysiological study. Using similar stimulus material as Fiebach, Schlesewsky, Lohmann, et al. (2005) and Fiebach, Schlesewsky, and Friederici (2001), this study investigated sentence processing in low span and high span participants by measuring ERPs (Fiebach, Schlesewsky, \& Friederici, 2002). In this study, the authors observed a sustained left frontal negativity between the wh-word and its trace which was larger for low than for high span readers. The brain imaging and electrophysiological studies together suggest that in the context of sentence reading, activation in BA 44 increases as a function of increasing syntactic working memory demands and, moreover, is dependent also upon the amount of working memory capacity available to the individual. The present study provides more direct evidence that BA 44 varies in its activation as a function of both the sentences' syntactic working memory demands and the individually available working memory capacity. That is, syntactic working memory demands as defined by word-order processing costs and by the distance over which the syntactic ambiguity in the sentence persisted were reflected in a higher activation in BA 44 for low span readers only.

The assumptions developed here regarding the relation of the pars opercularis of the IFG (i.e., BA 44) and syntactic working memory demands receive good support from patient studies. Lesions in Broca's aphasics usually include left BA 44 as the most prominent area (but see Caplan, Hildebrandt, \& Makris, 1996). These patients have been shown to display dramatic comprehension deficits when processing syntactically complex sentences (for an overview, see Grodzinsky, 2000). One important factor underlying these comprehension deficits is the reduction of working memory resources available for syntactic processing (e.g., Frazier \& Friederici, 1991). Thus, the present findings help to specify the exact function that the left BA 44 plays within the temporo-frontal network for sentence processing.

\section{The Right Hemispheric Involvement in Ambiguity Processing}

In addition to the mostly left-dominant network discussed above, the effect of the length of the ambiguous region (with larger activation for late as compared to early disambiguation in the superior portion of BA 44 
and the parietal lobe) was also observable in the right hemisphere. This finding suggests that the increased processing demands in syntactically ambiguous sentences lead to an additional involvement of the nondominant hemisphere. This view is also raised by Stowe, Paans, Wijes, and Zwarts (2004), who found activation in the right hemisphere for the processing of syntactic ambiguity. Additional imaging studies as well as lesion studies must demonstrate whether this interpretation of the right hemispheric involvement holds.

\section{Conclusion}

Within the temporo-frontal and subcortical network for language processing, the pars opercularis of the IFG (i.e., BA 44) appears to be the cortical area in which syntactic working memory demands and individually available working memory capacities interact during syntactic processing. The involvement of the right hemisphere during the processing of ambiguous sentences can be specified as follows: The right BA 44 and parietal areas become involved for all readers when syntactic ambiguity in a sentence is resolved late. Low span readers increase their activation in the right basal ganglia and thalamic structures in addition as a reflection of the greater effort in attempting to either keep two structures activated at the same time in working memory, or inhibit one of the two alternative readings. Taken together, it appears that the interactions of syntactic processing demands and individual processing resources observed in brain activation in BA 44 are directly related to the well-known behavioral observation that individuals with a low working memory capacity have more problems in comprehending syntactically difficult sentences.

\section{METHODS}

\section{Participants}

Sixteen participants took part in the experiment. One participant was excluded from data analysis due to excessive movements during the functional scanning session, leaving data from 15 individuals (mean age 25 years, age range 21-33, 10 men). All participants were native speakers of German, right-handed, with normal or corrected-to-normal vision. Handedness was assessed with an abridged German version of the Edinburgh Inventory (Oldfield, 1971). The participants were selected on the basis of individual working memory span scores from a German version of the Daneman and Carpenter (1980) reading span task. Two groups were formed: a low span group of eight participants with a maximum reading span of 3.0 (mean 2.625, range 2.0-3.0), and a high span group of seven participants with a minimum reading span of 4.5 (mean 5.14 , range 4.5-6.0).

\section{Materials}

The sentence material consisted of four types of temporarily ambiguous sentences. Examples are given in Table 1. Half of the sentences contained subject-relative clauses (SR) and half object-relative clauses (OR). Half of the sentences of each clause type were disambiguated early and the other half was disambiguated late. Thus, the four sentence versions correspond to the full crossing of the factors word order (SR or OR) and disambiguation point (early or late). Furthermore, half of the sentence material started with a singular noun, the other half with a plural noun. The factor Number (singular or plural) was included as a control-factor to make sure that the disambiguating auxiliary occurred equally often in singular and in plural form in each condition.

After each experimental sentence, a true-false verification statement was presented in order to determine whether the experimental sentence had been understood correctly. Verification sentences had a subjectverb-object structure. There were two versions, depending on which noun was presented first:

1. The $\langle$ noun 1$\rangle\langle$ verb $\rangle$ the $\langle$ noun 2$\rangle$.

2. The $\langle$ noun 2$\rangle\langle$ verb $\rangle$ the $\langle$ noun 1$\rangle$.

The past participle of the experimental sentence was changed into the past tense in the verification sentence. For example, the following two versions of a verification sentence correspond to the late-disambiguation sentence versions shown in Table 1 :

1. Die Sportlerin suchte die Trainerinnen. (The atblete looked for the trainers).

2. Die Trainerinnen suchten die Sportlerin. (The trainers looked for the atblete).

\section{Procedure}

Each trial had a duration of 22 sec. It started with the presentation of an asterisk for $700 \mathrm{msec}$, followed by a blank screen for $300 \mathrm{msec}$. Then the experimental sentence was presented word-by-word (300 msec visual presentation per word, $200 \mathrm{msec}$ ISI). Sentence length varied from 13 to 16 words due to the varying length of a final clause occurring after the critical manipulation [compare conditions (1a) and (2a) vs. (3a) and (4a) in Table 1]. After the presentation of the last word of the experimental sentence, a blank screen was presented for 500 msec. Next, the verification sentence was presented in one frame. Participants had to decide whether or not the content of this verification sentence agreed with the content of the previously presented sentence. If the content was the same, they had to press the yes-button with their right index finger, otherwise the no-button with their right middle finger. The verification sentence remained on the screen until the participant had responded, or disappeared automatically after $4000 \mathrm{msec}$. 
After the presentation of the verification sentence, a blank screen was presented for $100 \mathrm{msec}$, followed by visual feedback on sentence-verification performance. The feedback consisted of the word "correct" in green letters, the word "incorrect" in red letters, or the words "please faster" in red letters presented for $600 \mathrm{msec}$, followed by a blank screen of $300 \mathrm{msec}$. After this feedback, an ISI was used with variable length, but with a minimum length of $7.5 \mathrm{sec}$ to make the total trial length $22 \mathrm{sec}$. The actual length of the ISI of a particular trial was dependent on the length of the sentence, and on the speed of responding to the verification sentence. The words of the sentences were presented in black letters (proportional fonts) against a light gray background in the center of the screen. Stimuli were projected onto a translucent screen which the participants viewed via a mirror. The use of lowercase and uppercase letters conformed to the rules of German orthography. Participants were asked to read the sentences carefully, to lay still, and to respond as fast and accurately as possible. In order to familiarize with the task, participants were trained on a laptop computer outside of the magnet.

The construction and balancing of sentence materials was performed in the following way. A set of 128 basic sentence structures was derived from 128 different triplets of two nouns and a transitive verb. From every sentence in this set, eight versions were constructed by fully crossing the factors "word order" (SR/OR), "disambiguation point" (early/late), and "answer" (yes/no). Participants saw only one sentence from each set. We therefore constructed 8 different lists of 128 sentences, in which we also balanced the number of singular and plural sentences. Sentences were ordered pseudorandomly, with every condition equally often preceded by any of the other conditions.

\section{Analysis of Behavioral Data}

For the sentence-verification task, reaction times were measured from the onset of the verification sentence to the onset of the reaction of the participant. Trials that were incorrectly verified or answered too slowly were excluded from the behavioral analysis (as well as from the fMRI analysis). Reaction times deviating more than 2.5 standard deviations from the individual's mean were discarded to correct for outliers. Reaction times and percentages of correct responses were aggregated by participant and condition and then entered into an analysis of variance with the factors "word order" (SR/ OR) and "disambiguation point" (early/late) as withinsubject factors.

\section{fMRI Data Acquisition and Analysis}

We measured fMRI from eight axial slices, using a BOLDsensitive gradient-echo echo-planar imaging sequence. The lower border of the third slice was aligned to the
AC-PC line such that the peri-sylvian region was covered in all participants. Data were acquired using a 3-T Bruker Medspec 30/100 scanner (Bruker, Ettlingen, Germany). Functional EPI images were measured with a repetition time of TR $=2 \mathrm{sec}$, an echo time of TE $=30 \mathrm{msec}$, a flip angle $90^{\circ}$, acquisition bandwidth $100 \mathrm{kHz}$, and a data matrix of 64 by 64 with a field of view of $19.2 \mathrm{~cm}$, yielding an in-plane resolution of 3 by $3 \mathrm{~mm}$. Slice thickness was $5 \mathrm{~mm}$, with an interslice distance of $2 \mathrm{~mm}$. Prior to the functional measurement, T1-weighted anatomical MDEFT images (Ugurbil et al., 1993) (data matrix $256 \times$ 256, TR $1.3 \mathrm{sec}$, TE $10 \mathrm{msec}$ ) were obtained with a nonslice-selective inversion pulse followed by a single excitation of each slice (Norris, 2000). These anatomical images were used for coregistration of the functional data with high-resolution whole-head 3D MDEFT brain scans (128 sagittal slices, $1.5 \mathrm{~mm}$ thickness, FOV $25 \times$ $25 \times 19.2 \mathrm{~cm}$, data matrix $256 \times 256$ voxels; Lee et al., 1995; Ugurbil et al., 1993) which were acquired in a separate session.

Data analysis was performed using the LIPSIA software package (Lohmann et al., 2001; www.lipsia.org) which implements statistical methods established in the SPM99 software. Preprocessing comprised a movement correction, slicetime correction (using sinc-interpolation), as well as the application of a high-pass filter (cutoff frequency $88 \mathrm{sec}$ ) and a spatial smoothing using a gaussian kernel with $5.6 \mathrm{~mm}$ full width at half maximum (FWHM). The statistical evaluation was carried out by modeling the time interval between 3000 and $5500 \mathrm{msec}$ (i.e., 1.25 time steps) of the trial by a box-car function. The box-car function was convolved with a canonical hemodynamic response function as implemented in the SPM99 software, consisting of two summed gamma functions modeling a hemodynamic response function with a peak at around 5 sec and a late undershoot (e.g., Friston et al., 1998). The specified interval was chosen in order to account for the fact that the critical eventnamely, the point at which the temporally ambiguous sentences are disambiguated towards an SR or OR reading-is occurring at different time points in early and late disambiguated sentences. The epoch chosen spans the region between the two disambiguation points. Like this, it is unlikely that activation differences seen in association to the time point of disambiguation are due to the amount of sentence processing that took place before the disambiguation point. The epoch furthermore spans exactly that portion of the sentences for which differential working memory demands are predicted for early as compared to late disambiguated sentences. Incorrectly answered trials and timeouts were excluded from the statistical analysis.

Functional data were convolved with the predictor time series using a gaussian kernel of dispersion of $4 \mathrm{sec}$ FWHM. Contrast images reflecting the brain activation specifically evoked during processing of the critical sentence region were calculated for each individual by 
comparing the predictor time series with the experiment's mean activation level using a " 10 " contrast, where the first weight refers to the modeled regressor, and the second weight refers to the column of the design matrix that represents the overall mean of the time series. In a second-level analysis, individual contrast images were introduced as random effects together with regressors specifying the working memory span group. In this analysis, the main effect of both groups was examined in order to determine whether the activation at each voxel differed significantly from the overall mean, independent of span group. The resulting statistical $t$ maps were transformed into $Z$-values and thresholded using a combined voxel level criterion $(p<.001$, uncorrected for multiple comparisons) and cluster size criterion ( $p<.05$, corrected for multiple comparisons). In addition, a two-sample $t$ test was performed comparing activation elicited by low and high span individuals. $\operatorname{SPM}\{Z\}$ for the group comparison were thresholded at $p<.01$ (see, e.g., Pallier et al., 2003).

For a subsequent region-of-interest (ROI) statistics, we determined spherical ROIs (radius $3 \mathrm{~mm}$ ) based on the activation pattern seen in the above-described analysis. For each individual, ROI and sentence condition, we determined the mean contrast value; these values were entered into a repeated-measures analysis of variance using a standard statistical package. Brain regions showing bilateral activation were analyzed in a joint analysis, with the additional factor hemisphere. Altogether, 10 tests were performed, corresponding to the brain areas listed in Table 3. A corrected significance threshold was used in order to compensate for the fact that separate analyses of variance were performed for different brain regions (see, e.g., Chein \& Fiez, 2001, for a similar procedure). We applied a modified Bonferonni correction described by Keppel (1991, pp. 169-170) which yielded a corrected rejection probability of $\alpha=$ .02 for 10 separate tests.

\section{Acknowledgments}

We thank Stefan Zysset for his helpful comments analyzing the fMRI data and Susanne Wagner for help during preparation of the experiment. The present research was supported by the German Research Foundation (DFG) by research grant FI 848/1 awarded to CJF and ADF and by the Leibniz Research Prize awarded to ADF. It was conducted while Sandra Vos was a research fellow at the Max Planck Institute of Cognitive Neuroscience in Leipzig. Christian Fiebach is now at the Department of Psychology and Helen Wills Neuroscience Institute, University of California, Berkeley.

Reprint requests should be sent to Christian J. Fiebach, 132 Barker Hall, University of California, Berkeley, CA 94720-3190, or via e-mail: christian@fiebach.org.

The data reported in this experiment have been deposited in the fMRI Data Center (http://www.fmridc.org). The accession number is 2-2004-116ER.

\section{REFERENCES}

Ben-Shachar, M., Hendler, T., Kahn, I., Ben-Bashat, D., \& Grodzinsky, Y. (2003). The neural reality of syntactic transformations: Evidence from fMRI. Psychological Science, 14, 433-440.

Bergman, H., Feingold, A., Nini, A., Raz, A., Solvin, H., Abeles, M., \& Vaadia, E. (1998). Physiological aspects of information processing in the basal ganglia of normal and parkinsonian primates. Trends in Neuroscience, 21, 32-38.

Bookheimer, S. (2002). Functional MRI of language: New approaches to understanding the cortical organization of semantic processing. Annual Review of Neuroscience, 25, 151-188.

Caplan, D. (2001). Functional neuroimaging studies of syntactic processing. Journal of Psycholinguistic Research, 30, 297-320

Caplan, D., Alpert, N., \& Waters, G. (1998). Effects of syntactic structure and propositional number on patterns of regional blood flow. Journal of Cognitive Neuroscience, 10, 541-552.

Caplan, D., Alpert, N., \& Waters, G. (1999). PET studies of sentence processing with auditory sentence presentation. Neuroimage, 9, 343-351.

Caplan, D., Alpert, N., Waters, G., \& Olivieri, A. (2000). Activation of Broca's area by syntactic processing under conditions of concurrent articulation. Human Brain Mapping, 9, 65-71.

Caplan, D., Hildebrandt, N., \& Makris, N. (1996). Location of lesions in stroke patients with deficits in syntactic processing in sentence comprehension. Brain, 119, 933-949.

Caplan, D., Vijayan, S., Kuperberg, G., West, C., Waters, G., Greve, D., \& Dale, A. M. (2002). Vascular responses to syntactic processing: Event-related fMRI study of relative clauses. Human Brain Mapping, 15, 26-38.

Caplan, D., \& Waters, G. (1999). Online syntactic processing in aphasia: Studies with auditory moving window presentation. Brain and Language, 69, 330-333.

Chein, J. M., \& Fiez, J. A. (2001). Dissociation of verbal working memory system components using a delayed serial recall task. Cerebral Cortex, 11, 1003-1014.

Cooke, A., Zurif, E. B., DeVita, C., Alsop, D., Koenig, P., Detre, J., Gee, J., Pinango, M., Balogh, J., \& Grossman, M. (2001). Neural basis for sentence comprehension: Grammatical and short-term memory components. Human Brain Mapping, 15, 80-94.

Copland, D. A., Chenery, H. J., \& Murdoch, B. E. (2001). Discourse priming of homophones in individuals with dominant nonthalamic subcortical lesions, cortical lesions and Parkinson's Disease. Journal of Clinical and Experimental Neuropsychology, 23, 538-556.

Daneman, M., \& Carpenter, P. A. (1980). Individual differences in working memory and reading. Journal of Verbal Learning and Verbal Behavior, 19, 450-466.

Daneman, M., \& Merikle, P. M. (1996). Working memory and language comprehension: A meta-analysis. Psychonomic Bulletin and Review, 3, 422-433.

Dapretto, M., \& Bookheimer, S. Y. (1999). Form and content: Dissociating syntax and semantics in sentence comprehension. Neuron, 24, 427-432.

Embick, D., Marantz, A., Miyashita, Y., O’Neil, W., \& Sakai, K. L. (2000). A syntactic specialization for Broca's area. Proceedings of the National Academy of Sciences, U.S.A., 97, 6150-6154.

Fiebach, C. J., Schlesewsky, M., \& Friederici, A. D. (2001). Syntactic working memory and the establishment of filler-gap dependencies: Insights from ERPs and fMRI. Journal of Psycholinguistic Research, 30, 321-338. 
Fiebach, C. J., Schlesewsky, M., \& Friederici, A. D. (2002). Separating syntactic memory costs and syntactic integration costs during parsing: The processing of German WH-questions. Journal of Memory and Language, 47, 250-272.

Fiebach, C. J., Schlesewsky, M., Lohmann, G., von Cramon, D. Y., \& Friederici, A. D. (2005). Revisiting the role of Broca's area in sentence processing: Syntactic integration vs. syntactic working memory. Human Brain Mapping, 24, 79-91.

Frazier, L. (1987). Sentence processing: A tutorial review. In: M. Coltheart (Ed.), Attention and performance: Vol. XII (pp. 559-586). Hillsdale, NJ: Erlbaum.

Frazier, L., \& Friederici, A. D. (1991). On deriving the properties of agrammatic comprehension. Brain and Language, 40, 51-66.

Friederici, A. D. (2002). Towards a neural basis of auditory sentence processing. Trends in Cognitive Sciences, 6, 78-84.

Friederici, A. D., Kotz, S. A., Werheid, K., Hein, G., \& von Cramon, D. Y. (2003). Syntactic comprehension in Parkinson's disease: Investigating early automatic and late integrational processes using event-related brain potentials. Neuropsychology, 17, 133-142.

Friederici, A. D., Meyer, M., \& von Cramon, D. Y. (2000). Auditory language comprehension: An event-related fMRI study on the processing of syntactic and lexical information. Brain and Language, 74, 289-300.

Friederici, A. D., Rueschemeyer, S.-A., Hahne, A., \& Fiebach, C. J. (2003). The role of left inferior frontal and superior temporal cortex in sentence comprehension: Localizing syntactic and semantic processes. Cerebral Cortex, 13, 170-177.

Friederici, A. D., Steinhauer, K., Mecklinger, A., \& Meyer, M. (1998). Working memory constraints on syntactic ambiguity resolution as revealed by electrical brain responses. Biological Psychology, 47, 193-221.

Friston, K. J., Fletcher, P., Josephs, O., Holmes, A., Rugg, M. D., \& Turner, R. (1998). Event-related fMRI: Characterizing differential responses. Neuroimage, 7, 30-40.

Grodzinsky, Y. (2000). The neurobiology of syntax: Language use without Broca's area. Behavioral and Brain Science, 23, $1-17$.

Grossman, M., Carvell, S., Gollomp, S., Stern, M. B., Vernon, R. N., \& Hurtig, H. I. (1991). Sentence comprehension and praxis deficits in Parkinson's disease. Neurology, 41, 1620-1026.

Gunter, T. C., Wagner, S., \& Friederici, A. D. (2003). Working memory and lexical ambiguity resolution as revealed by ERPs: A difficult case for activation theories. Journal of Cognitive Neuroscience, 15, 643-657.

Humphries, C., Kimberley, T., Buchsbaum, B., \& Hickok, G. (2001). Role of anterior temporal cortex in auditory sentence comprehension: An fMRI study. NeuroReport, 12, 1749-1752.

Just, M. A., \& Carpenter, P. A. (1992). A capacity theory of comprehension: Individual differences in working memory. Psychological Review, 99, 122-149.

Just, M. A., Carpenter, P. A., Keller, T. A., Eddy, W. F., \& Thulborn, K. R. (1996). Brain activation modulated by sentence comprehension. Science, 274, 114-116.

Kaan, E., \& Swaab, T. Y. (2002). The brain circuitry of syntactic comprehension. Trends in Cognitive Sciences, 6, 350-356.

Kapur, S., Craik, F. I. M., Tulving, E., Wilson, A. A., Houle, S., \& Brown, G. M. (1994). Neuroanatomical correlates of encoding in episodic memory: Levels of processing effect. Proceedings of the National Academy of Sciences, U.S.A., 91, 2008-2011.
Keppel, G. (1991). Design and analysis: A researcher's handbook (3rd ed.). Englewood Cliffs, NJ: Prentice-Hall.

Kotz, S. A., Cappa, S. F., von Cramon, D. Y., \& Friederici, A. D. (2002). Modulation of the lexical-semantic network by auditory semantic priming: An event-related functional MRI study. Neuroimage, 17, 1761-1772.

Kotz, S. A., Frisch, S., von Cramon, D. Y., \& Friederici, A. D. (2003). Syntactic language processing: ERP lesion data on the role of the basal ganglia. Journal of the International Neuropsychological Society, 9, 1053-1060.

Kuperberg, G. R., McGuire, P. K., Bullmore, E. T., Brammer, M. J., Rabe-Hesketh, S., Wright, I. C., Lythgoe, D. J., Williams, S. C. R., \& David, A. S. (2000). Common and distinct neural substrates for pragmatic, semantic, and syntactic processing of spoken sentences: An fMRI study. Journal of Cognitive Neuroscience, 12, 321-341.

Lee, J.-H., Garwood, M., Menon, R., Adriany, G., Andersen, P., Truwit, C. L., \& Ugurbil, K. (1995). High contrast and fast three-dimensional magnetic resonance imaging at high fields. Magnetic Resonance in Medicine, 34, 308-312.

Lohmann, G., Müller, K., Bosch, V., Mentzel, H., Hessler, S., Chen, L., \& von Cramon, D. Y. (2001). LIPSIA-A new software system for the analysis of functional magnetic resonance images of the human brain. Computerized Medical Imaging and Graphics, 25, 449-457.

Luke, K. K., Liu, H.-L., Wan, Y.-L., \& Tan, L. H. (2002). Functional anatomy of syntactic and semantic processing in language comprehension. Human Brain Mapping, 16, $133-145$.

MacDonald, M. C., Just, M. A., \& Carpenter, P. A. (1992). Working memory constraints on the processing of syntactic ambiguity. Cognitive Psychology, 24, 56-98.

Mecklinger, A., Schriefers, H., Steinhauer, K., \& Friederici, A. D. (1995). Processing relative clauses varying on syntactic and semantic dimensions: An analysis with event-related potentials. Memory and Cognition, 23, 477-494.

Meyer, M., Friederici, A. D., \& von Cramon, D. Y. (2000). Neurocognition of auditory sentence comprehension: Event-related fMRI reveals sensitivity to syntactic violations and task demands. Cognitive Brain Research, 9, 19-33.

Mitchell, D. C. (1994). Sentence parsing. In M. A. Gernsbacher (Ed.), Handbook of psycholinguistics (pp. 375-409). San Diego: Academic Press.

Moro, A., Tettamanti, M., Perani, D., Donati, C., Cappa, S. F., \& Fazio, F. (2001). Syntax and the brain: Disentangling grammar by selective anomalies. Neuroimage, 13, 110-118.

Ni, W., Constable, R. T., Mencl, W. E., Pugh, K. R., Fulbright, R. K., Shaywitz, S. E., Shaywitz, B. A., Gore, J. C., \& Shankweiler, D. (2000). An event-related neuroimaging study distinguishing form and content in sentence processing. Journal of Cognitive Neuroscience, 12, 120-133.

Norris, D. G. (2000). Reduced power multislice MDEFT imaging. Journal of Magnetic Resonance Imaging, 11, 445-451.

Oldfield, R. C. (1971). The assessment and analysis of handedness: The Edinburgh Inventory. Neuropsychology, 9, 97-113.

Pallier, C., Dahaene, S., Poline, J. B., LeBihan, D., Argenti, A. M., Dupoux, E., \& Mehler, J. (2003). Brain imaging of language plasticity in adopted adults: Can a second language replace the first? Cerebral Cortex, 13, 155-161.

Poldrack, R. A., Wagner, A. D., Prull, M. W., Desmond, J. E., Glover, G. H., \& Gabrieli, J. D. (1999). Functional specialization for semantic and phonological processing in the left inferior prefrontal cortex. Neuroimage, 10, $15-35$. 
Pugh, K. R., Shaywitz, B. A., Shaywitz, S. E., Constable, R. T., Skudlarski, P., Fulbright, R. K., Bronen, R. A., Shankweiler, D. P., Katz, L., Fletcher, J. M., \& Gore, J. C. (1996). Cerebral organization of component processes in reading. Brain, 119, 1221-1238.

Röder, B., Stock, O., Neville, H., Bien, S., \& Rösler, F. (2002). Brain activation modulated by the comprehension of normal and pseudo-word sentences of different processing demands: A functional magnetic resonance imaging study. Neuroimage, 15, 1003-1014.

Schriefers, H., Friederici, A. D., \& Kuehn, K. (1995). The processing of locally ambiguous relative clauses in German. Journal of Memory and Language, 34, 499-520.

Scott, S. K., Blank, C. C., Rosen, S., \& Wise, R. J. S. (2000). Identification of a pathway for intelligible speech in the left temporal lobe. Brain, 123, 2400-2406.

Smith, E. E., \& Jonides, J. (1999). Storage and executive processes in the frontal lobes. Science, 283, 1657-1661.

Stowe, L. A., Paans, A. M. J., Wijers, A. A., \& Zwarts, F. (2004). Activations of "motor" and other non-language structures during sentence comprehension. Brain and Language, 89, 290-299.

Stromswold, K., Caplan, D., Alpert, N., \& Rauch, S. (1996). Localization of syntactic comprehension by positron emission tomography. Brain and Language, 52, 452-473.

Suzuki, K., \& Sakai, K. L. (2003). An event-related fMRI study of explicit syntactic processing of normal/anomalous sentences in contrast to implicit syntactic processing. Cerebral Cortex, 13, 517-526.
Talairach, J., \& Tournoux, P. (1988). Co-planar stereotaxis atlas of the buman brain. New York: Thieme Medical.

Ugurbil, K., Garwood, M., Ellermann, J., Hendrich, K., Hinke, R., Hu, X., Kim, S. G., Menon, R., Merkle, H., Ogawa, S., \& Salmi, R. (1993). Imaging at high magnetic fields: Initial experiences at 4 T. Magnetic Resonance Quarterly, 9, 259-277.

Ullman, M. T., Corkin, S., Coppola, M., Hickok, G., Growdon, J. H., Koroshetz, W. J., \& Pinker, S. (1997). A neural dissociation within language: Evidence that the mental dictionary is part of declarative memory, and that grammatical rules are processed by the procedural system. Journal of Cognitive Neuroscience, 9, 266-276.

Vos, S. H., Gunter, T. C., Kolk, H. H. J., \& Mulder, G. (2001). Working memory constraints on syntactic processing: An electrophysiological investigation. Psychophysiology, 38, 41-64.

Vos, S. H., Gunter, T. C., Schriefers, H., \& Friederici, A. D. (2001). Syntactic parsing and working memory: The effects of syntactic complexity, reading span, and concurrent load. Language and Cognitive Processes, 16, 65-103.

Waters, G., Caplan, D., Alpert, N., \& Stanczak, L. (2003). Individual differences in rCBF correlates of syntactic processing in sentence comprehension: Effects of working memory and speed of processing. Neuroimage, 19, 101-112.

Waters, G. S., \& Caplan, D. (1996). The measurement of verbal working memory capacity and its relation to reading comprehension. Quarterly Journal of Experimental Psychology, 1, 51-79. 